\title{
Isolation and Antimicrobial Susceptibility of Salmonella Typhimurium and Salmonella Enteritidis in Fecal Samples from Animals
}

\section{López-Martín $\mathrm{JI}^{1^{*}}$, González-Acuña $\mathrm{D}^{2}$, García $\mathrm{CA}^{3}$, and Carrasco LO}

${ }^{1}$ Department of Pathology and Preventive Medicine, Faculty of Veterinary Sciences, University of Concepcion, Concepcion, Chile

${ }^{2}$ Department of Animal Science, Faculty of Veterinary Sciences, University of Concepcion, Concepcion, Chile

${ }^{3}$ Department of Microbiology, Faculty of Biological Sciences, University of Concepcion, Concepcion, Chile

${ }^{4}$ Department of Anatomy and Comparative Pathology, Faculty of Veterinary Medicine, Córdoba, Spain

\begin{abstract}
Salmonella enterica is a microorganism with high zoonotic potential, distributed worldwide, having more than 2,610 identified serovars and affecting different animal species, both production and wildlife animals.

Objective: Prevention of human salmonellosis requires prior monitoring of Salmonella in animals. In this study. Salmonella enterica serovars from different animal origins were isolated and their antimicrobial susceptibility was determined.

Methods: A total of 2193 samples from different origins (feces of cattle, sheep, horses, pigs, dog, chickens and seagulls and chicken eggs) were analyzed for bacterial typing and antimicrobial susceptibility was studied by the Kirby-Bauer method including 9 antibacterial agents (florfenicol, amoxicillin, ceftiofur, gentamicin, oxytetracycline, trimethoprim/sulfamethoxazole, enrofloxacine, ampicillin and cefoperazone) and $\mathrm{MIC}_{50}$ and $\mathrm{MIC}_{90}$ for 6 of them were determined.

Results: A 4.38\% serovar isolation $(n=96)$, including 6 S. Typhimurium $(6.25 \%)$ from equine cattle and pig feces, 19 S. Enteritidis (19.79\%) from seagulls and pigs and 71 other serovars, was obtained from the various animal origins. Serovar S. Typhimurium showed high resistance to oxytetracycline and gentamicin by the Kirby-Bauer method and a $\mathrm{MIC}_{90}$ of $512 \mu \mathrm{g} \cdot \mathrm{mL}^{-1}$ for oxytetracycline and trimethoprim/sulfamethoxazole antibiotics. By the diffusion method, serovar $S$. Enteritidis was resistant to trimethoprim/sulfamethoxazole and its $\mathrm{MIC}_{90}$ was $256 \mu \mathrm{g} \cdot \mathrm{mL}^{-1}$ for oxytetracycline. The $32 \%$ of Salmonella isolates showed multi-resistance, 2 strains, isolated from pigs (one S. Typhimurium and one $S$. Enteritidis serovars), showed resistance to 5 antimicrobials tested.
\end{abstract}

Conclusion: The constant release of these serovars to the environment, reaching also animal food, is a permanent potential risk for public health, turning into a first priority the establishment of control and antibiotic therapy strategies.

Keywords: Salmonella enterica; S. typhymurium; S. Enteritidis; Antimicrobial; Salmonellosis; $\mathrm{MIC}_{50}$ MIC $_{90}$

\section{Introduction}

Different serovars of Salmonella enterica subspecies enterica are potentially zoonotic pathogens. Different animal species, distributed throughout the world, have been detected as carriers of this pathogenic agent [1]. More than 2610 S. enterica serovars have been recognized worldwide, most of them being major causative agents of diseases in humans and animals, producing gastroenteritis and other acute infections [2]. In the United States, it is estimated that, in humans, approximately $44 \%$ of hospitalizations, $44 \%$ of deaths and $20 \%$ of illnesses are the consequence of foodborne pathogens [3], representing an incidence of 15.2 cases per 100,000 individuals [4].

Salmonella is a persistent pathogen in the environment, able to easily survive and proliferate [1]. The most commonly isolated serovars worldwide from various animal sources continue to be $S$. Enteritidis and $S$. Typhimurium which, besides producing gastroenteritis, are found in asymptomatic carriers in a wide variety of animal species [58]. Of these, $S$. Enteritidis it is the most prevalent one followed by $S$. Typhimurium (52.3\% and $23.3 \%$ of the cases, respectively) [9].

Non-typhoid salmonellosis in humans are mainly caused by contamination of foods from animal origin $[8,10,11]$. Human infections with $S$. Enteritidis originate mainly from eggs and egg products when consumed raw or undercooked, while $S$. Typhimurium infections originate mainly from pigs, cattle and chicken as well as environmental contamination from household pets or contaminated birds $[1,11]$.
In general, approximately $95 \%$ of human cases of salmonellosis are associated to the consumption of contaminated products such as meat, chicken, eggs, milk, seafood and fresh produce [12]. In China, Salmonella serovars were isolated from 54\% of chicken samples, $31 \%$ of pig samples, $17 \%$ of cattle samples and $20 \%$ of sheep samples [7]. In northern Vietnam, where only pig and chicken samples were obtained, the frequency of Salmonella serovars isolation was $39.6 \%$ and $42.9 \%$, respectively [13]. With respect to eggs, $4.82 \%$ were positive for Salmonella [14]. On the other hand, 2 (1.33\%) of 150 eggshell samples were determined as contaminated with Salmonella, including both $S$. Enteritidis and $S$. Typhimurium [15]. The serovar most commonly identified in chicken meat is the serovar $S$. Enteritidis, also reported as the most common in human cases of salmonellosis [7]; however, other authors reported serovar $S$. Typhimurium as the most common [14].

*Corresponding author: López-Martín Juana Isabel, Avda. Vicente Méndez 595 Chillán Casilla 537, Chile, Código postal: 3780000, Tel: 5642220 8822, Fax: 56 42 2273201; E-mail: jlopez@udec.cl

Received January 25, 2016; Accepted March 16, 2016; Published March 25 , 2016

Citation: López-Martín JI, González-Acuña D, García CA, Carrasco LO (2016) Isolation and Antimicrobial Susceptibility of Salmonella Typhimurium and Salmonella Enteritidis in Fecal Samples from Animals. J Antimicro 2: 109. doi:10.4172/2472-1212.1000109

Copyright: ( 2016 López-Martín Jl, et al. This is an open-access article distributed under the terms of the Creative Commons Attribution License, which permits unrestricted use, distribution, and reproduction in any medium, provided the original author and source are credited. 
In the United States, during the years $1973-2011,5 \%$ of outbreaks of salmonellosis were due to consumption of meat products [10].

In addition, in a review, was reported that a number of marine animals and fish eating birds are reservoirs of $S$. enterica serovars without showing any clinical manifestation of the disease [16]. In general, seagulls have been involved in major outbreaks of salmonellosis in animals and in humans [17].

However, the predominant serovars vary not only according to the species affected, but also among different geographical areas of a region [8].

It is known that intestinal salmonellosis can be a self-limited disease not requiring antibiotic therapy [5]. However, bacteremia may appear in young or old individuals in humans or various other animal species and also in immunocompromised individuals. Thus, transference of antibiotic resistance from environmental to human pathogenic bacteria is considered a major risk for public health. Therefore, the presence of antibiotic resistant bacteria, mainly in fecal contamination, plays an important role in the spread of these resistance by several mechanisms, including gene transfer [18]. In 2003, was reported that $22.5 \%$ of nontyphoid Salmonella in humans were resistant to at least 1 antimicrobial agent [12]. Recently, was indicated that in isolates from children in Kenya, $97 \%$ of $S$. Typhimurium and $92 \%$ of $S$. Enteritidis showed resistance to at least one antimicrobial agent [19]. Multidrug resistance (MDR) has been reported for $S$. Typhimurium isolated from bovines and porcines in Japan where $82 \%$ of the samples showed resistance to at least one antibiotic and $70 \%$ to three or more antibacterial agents [6]. Also, both healthy animal samples and clinical samples from different species showed that $44 \%$ of Salmonella isolates were resistant to at least one antibacterial agent, and $4.8 \%$ of these isolates showed the same resistance phenotype previously described for humans [12]. Moreover, $90.1 \%$ of $S$. Enteritidis resistant strains isolated from various human and animal origins were resistant to at least one antibacterial in Brazil [20] and nearly $80 \%$ of Salmonella isolates from chicken, pig, cattle and lamb meat were resistant to at least one antimicrobial while $53 \%$ of them were resistant to more than three antimicrobial agents [7]. Rodriguez-Rivera et al. isolated Salmonella from bovine feces and from the environment in farms showing a 23.6\% resistance from 1 to 11 antimicrobial agents, representing 50 different antibacterial resistance patterns, and concluded that it represents a potential risk for public health [21].

The most common MDR was to ampicillin, chloramphenicol, streptomycin, sulfamethoxazole and tetracycline $[6,19,22]$. The indiscriminated use of these compounds makes difficult the treatment of bacterial infections in animals and humans [23].

For all the above, there is no doubt that prevention of human salmonellosis requires prior monitoring of Salmonella from animal origin. Therefore, the aims of this study were to isolate, identify, serotyping and to determine the antimicrobial susceptibility of $S$. enterica strains isolated from fecal samples of different animal species to obtain data to contribute to the control and to determine the dissemination of Salmonella serovars. Results reported here include, in part, the genotypic and phenotypic characterization of Salmonella isolates from different animals origin.

\section{Materials and Methods}

\section{Samples}

The Veterinary Microbiology Diagnostic Laboratory, Department of Pathology and Preventive Medicine, Faculty of Veterinary Sciences, University of Concepcion, Chile (MedVet-UdeC Laboratory) received fecal swab samples from horses (47), pigs (326) and cattle (72) with gastroenteritis. In addition, fecal samples of dogs from the Veterinary Clinical Hospital, Faculty of Veterinary Sciences, University of Concepción and from stray dogs (258) plus sheep samples (131) were analyzed. Research projects also provided egg samples (444), fecal swabs of backyard chickens (609) and fecal swabs of seagulls from the port of Talcahuano, Chile (306), collected by statistical random system. In total, 2193 samples of diverse animal sources were obtained and analyzed searching for the presence of Salmonella enterica serovars.

\section{Isolation and identification of Salmonella enterica}

The conventional bacteriological method of the MedVet-UdeC Laboratory was used. Fecal swab samples were subjected to an initial pre-enrichment in buffered peptone water (Merck) and incubated for 24 hours at $37^{\circ} \mathrm{C}$ and $0.5 \mathrm{~mL}$ were transferred to $10 \mathrm{~mL}$ Tetrathionate Broth (Merck) and incubated at $42^{\circ} \mathrm{C}$ for $24 \mathrm{~h}$. Finally, a loopful was transferred to dishes containing XLD agar (Merck), a Salmonella selective medium, in triplicate, and incubated for $18 \mathrm{~h}$ at $37^{\circ} \mathrm{C}$. Presumptive Salmonella colonies were phenotypically confirmed by biochemical properties in differential agars, such as TSI, LIA, Simmons citrate and SIM (Merck) and incubated at $37^{\circ} \mathrm{C}$ for $24-48 \mathrm{hrs}$. Colonies biochemically confirmed as $S$. enterica were subjected to serological confirmation by agglutination with polyvalent antiserum O (Denka Senken Co. ltda). S. Typhimurium and S. Enteritidis, ATCC 14028 and 13076, respectively, were used as positive controls. After biochemistry and serology tests confirmed the identification of S. enterica, the strains were sent to the National Reference Laboratory, Public Health Institute (ISP), Chile for serotyping. The serovars isolates were stored to $20^{\circ} \mathrm{C}$ and constitute part of the culture collection of the MedVet-UdeC Laboratory.

\section{Antimicrobial susceptibility}

The qualitative agar diffusion method (Kirby-Bauer method) was used employing Mueller Hinton agar. Nine antibacterial agents Florfenicol (FLO) $30 \mu \mathrm{g}$, amoxicillin (AMX) $20 \mu \mathrm{g}$, ceftiofur (CLR) $30 \mu \mathrm{g}$, gentamicin (GEN) $10 \mu \mathrm{g}$, oxytetracycline (TET) $30 \mu \mathrm{g}$, trimethoprim/sulfamethoxazole (SXT) $25 \mu \mathrm{g}$, enrofloxacin (EEE) $30 \mu \mathrm{g}$, ampicillin (AMP) $10 \mu \mathrm{g}$ and Cefoperazone (CEF) $75 \mu \mathrm{g}$ were used. The antimicrobial susceptibility was determined according to the standard diameter of inhibition ( $\mathrm{mm}$ ) for each antibiotic used [24]. Minimun Inhibitory Concentrations (MIC) were determined by the serial dilution method in Mueller Hinton broth for 6 of the 9 antibiotics used: AMP, TET, SXT, FLO, EEE and CLR. A $1024 \mu \mathrm{g} \mathrm{mL-1} \mathrm{stock}$ solution was obtained for each antibiotic [24]. Incubation was carried out at $37^{\circ} \mathrm{C}$ for 24 hours. Control strains were E. coli ATCC 25922 and S. enterica ATCC 31194 . The MIC $\left(\mathrm{MIC}_{50}\right.$ and $\left.\mathrm{MIC}_{90}\right)$ was determined for each of the antibiotics used and the sensitivity or resistance was determined according to the protocol described [24].

\section{Results}

\section{Bacterial strains}

Ninety-six strains of S. enterica were obtained, representing a $4.38 \%$ isolation rate. Of these, 6 corresponded to $S$. Typhimurium (6.25\%), 19 to $S$. Enteritidis (19.79\%), and the remaining 71 strains corresponded to other serovars (Table 1). S. Typhimurium serovars were obtained from clinical samples from pigs, ruminants and equines. With respect to $S$. Enteritidis serovars, 17 were obtained from cloacal samples of seagulls 


\begin{tabular}{|c|c|c|c|c|c|c|c|}
\hline & Bovine & Ovine & Porcine & Equine & Gull & $\begin{array}{c}\text { Egg } \\
\text { (chicken) }\end{array}$ & $\mathbf{n =}$ \\
\hline $\begin{array}{c}\text { S } \\
\text { Typhimurium }\end{array}$ & 2 & 0 & 2 & 2 & 0 & 0 & 6 \\
\hline S. anatum & 1 & 0 & 1 & 0 & 5 & 0 & 7 \\
\hline S. Enteritidis & 0 & 0 & 2 & 0 & 17 & 0 & 19 \\
\hline S. infantis & 0 & 0 & 24 & 0 & 13 & 1 & 38 \\
\hline S. selftenberg & 0 & 1 & 0 & 0 & 19 & 2 & 22 \\
\hline S. derby & 0 & 0 & 2 & 0 & 0 & 2 & 4 \\
\hline n= & 3 & 1 & 31 & 2 & 54 & 5 & 96 \\
\hline
\end{tabular}

Table1: Frequency of isolation of Salmonella enterica serovars from different origins.

(Larus dominicanus) and 2 were isolated from pig clinical samples.

From the 258 canine fecal samples and 609 chicken cloacal swabs from chickens (609) no S. enterica isolates were obtained. The $17.65 \%$ of $S$. enterica isolates were obtained from cloacal samples of seagulls, followed by porcine fecal samples $(9.51 \%)$.

\section{Antimicrobial susceptibility}

According to the Kirby-Bauer method for antibiotic susceptibility, $S$. Typhimurium and $S$. Enteritidis serovars isolated from different sources showed a high resistance to ampicillin, followed by trimethoprim/ sulfamethoxazole and then gentamicin and oxytetracycline (Table 2). $S$. Typhimurium isolates $(\mathrm{n}=6)$ showed a high resistance to oxytetracycline and gentamicin (50\%); however, a higher resistance to trimethoprim/ sulfamethoxazole (31.6\%) was observed in S. Enteritidis serovars (Table 2).

Table 3 shows the results of resistance of the $S$. Typhimurium and $S$. Enteritidis serovars according to the $\mathrm{MIC}_{50}$ and $\mathrm{MIC}_{90}$. The highest resistance was obtained for oxytetracycline and trimethoprim/ sulfamethoxazole. S. Enteritidis serovars were mainly resistant to tetracyclines but $S$. Typhimurium serovars showed a high MDR to tetracycline, trimethoprim/sulfamethoxazole, amoxicillin and florfenicol. In general, Salmonella isolates showed high sensitivity to cephalosporins and fluoroquinolones.

Table 4 shows the patterns of resistance. A $32 \%$ of the serovars isolated showed MDR. Among them, 6 S. Typhimurium serovars and the $2 \mathrm{~S}$. Enteritidis serovars were obtained from pig clinical samples. Strains showing MDR to 5 antibiotics were both isolated from pigs, one being $S$. Typhimurium and other $S$ Enteritidis.

\section{Discussion}

In the present study, the highest percentage of Salmonella spp. isolates were identified as $S$. infantis serovar and they were collected from pigs and seagulls and it was followed by $S$. Enteritidis and $S$. senftenberg serovars, also obtained from seagulls. The diversity of serovars isolated from the samples of different animal species confirms the different source of contamination, making it even more interesting to study the different possible sources of contamination considering the diversity of carrier individuals. In general, there is a high consistency between the literature reports and the findings of the present work. Thus, researchers of both human health and animal health should take appropriate precautions when working with Salmonella due to its different zoonotic potential and its role in public health, particularly when dealing with wildlife. A previous work had already reported the role of seagulls residing in the Chilean coast as potential vectors of pathogens multiresistant to antimicrobials with the ensuing risk for public and animal health [25].

\begin{tabular}{|c|c|c|c|c|c|c|c|c|c|}
\hline & AMP & EEE & CLR & SXT & GEN & TET & FLO & CEF & AMX \\
\hline $\begin{array}{c}\text { S. Typhimurium } \\
(n=6)\end{array}$ & 100 & 0 & 0 & 33 & 50 & 50 & 0 & 17 & 0 \\
\hline $\begin{array}{c}\text { S. Enteritidis } \\
(n=19)\end{array}$ & 84 & 5.3 & 11 & 32 & 16 & 11 & 0 & 0 & 0 \\
\hline Ambos $(n=25)$ & 88 & 4 & 8 & 32 & 24 & 20 & 0 & 4 & 0 \\
\hline
\end{tabular}

AMP: Ampicillin; EEE: Enrofloxacin; CLR: Ceftiofur; SXT: Trimethoprim/ sulfamethoxazole; GEN: Gentamicin; TET: Oxytetracyclin; FLO: Florfenicol; CEF Cefoperazone; AMX: Amoxicillin.

Table 2: Percentage distribution of antibiotic resistance, according to the KirbyBauer technique, for $S$. Typhimurium and $S$. Enteritidis serovars isolated from different animal sources.

A 2003 study found high similarity between strains of $S$. Enteritidis isolated from seagulls and strains from cattle, wild birds, pigs, horses and samples from food plants [26], demonstrating not only the spread of this pathogen by these birds but also the importance of these infections in animal production and in public health through food contamination. The biggest problem is caused by the spreading of this or other bacteria to animals, grasslands, food plants or water sources and through them they can reach other wild species which, in turn, could disseminate agents potentially pathogenic for humans and production animal species. Similar to what was found in this study, some authors point out that $63 \%$ of $S$. Enteritidis originates from birds and $90.8 \%$ of $S$. Typhimurium do it from porcines [11], being $S$. derby the most frequently reported in pigs [22]. In addition, the same authors mention that $42.4 \%$ of isolates from eggs are the source of human salmonellosis in Europe, followed by pigs (31.1\%) and chickens and turkeys being the least important source of salmonellosis [11]. In 2012, a prevalence of $7.2 \%$ of positive pigs and $52.6 \%$ of pig farms was reported [22]. It was previously reported, in equines, a $71 \%$ isolation of $S$. Typhimurium and only $8 \%$ isolation of $S$. Enteritidis [27]; however, the dissemination of Salmonella from this animal species to humans has not been reported. The same can be mentioned for S. enterica serovars from canines whose transmission to humans is unknown. No S. enterica serovars were found in the canine fecal samples analyzed in this work.

Antibiotic resistance in Salmonella isolates from samples of food or water sources is of great importance for public health. Considering that these sources of infection arise from animal feces, it is not less important to consider that antimicrobial agents can be used to treat or prevent infections and also as development promoters [28]. There are many reports indicating a high percentage of Salmonella isolates, from healthy and sick animals, resistant to two or more antimicrobial agents [29], explaining the high spread of these serovars through the feces of clinically healthy animals and their wide dissemination in the environment. The release of $S$. Typhimurium in a pig plant was reported [30]. This release was very high for several days post inoculation, even in animals demonstrating to be healthy, so even normal feces could be a source of within-herd infection. It has been found that multiresistant S. Enteritidis (resistant to two or more agents) can reach up to $51.6 \%$ with 18 different patterns [20]. The resistant patterns most commonly found are, in general, to sulfasoxazole, streptomycin and tetracycline while ciprofloxacin resistance was the least common. Pigs have a high frequency of antibiotic resistance in Salmonella serovars [6], similar to that found in the present work. In Japan, $S$. Typhimurium isolated from various types of animals showed that $20 \%$ of the isolates were resistant to ampicillin and $24 \%$ to tetracyclines [29]. In 2008, 
Citation: López-Martín JI, González-Acuña D, García CA, Carrasco LO (2016) Isolation and Antimicrobial Susceptibility of Salmonella Typhimurium and Salmonella Enteritidis in Fecal Samples from Animals. J Antimicro 2: 109. doi:10.4172/2472-1212.1000109

Page 4 of 6

\begin{tabular}{|c|c|c|c|c|c|c|c|c|c|c|c|c|c|c|c|c|c|}
\hline \multicolumn{18}{|c|}{ Serovares Typhimurium and Enteritidis } \\
\hline & MIC & & & & & & & & & & & & & & $\% R$ & $\mathrm{MIC}_{50}$ & $\mathrm{MIC}_{90}$ \\
\hline & $<0.5$ & 0.5 & 1 & 2 & 4 & 8 & 16 & 32 & 64 & 128 & 256 & 512 & 1024 & $>1024$ & & $\mu \mathrm{g} / \mathrm{mL}$ & $\mu \mathrm{g} / \mathrm{mL}$ \\
\hline EEE & 17 & 4 & 1 & 2 & 1 & & & & & & & & & & 12 & $<0.5$ & 2 \\
\hline TET & & & & 2 & 7 & 5 & 3 & 3 & & 1 & 2 & 2 & & & 32 & 8 & 256 \\
\hline SXT & & & & 1 & 3 & 4 & & 4 & 5 & & 2 & 5 & 1 & & 32 & 64 & 512 \\
\hline CLR & 5 & & 2 & 18 & & & & & & & & & & & 0 & 2 & 2 \\
\hline AMP & & & 14 & 5 & & 3 & & & & 2 & & 1 & & & 12 & 1 & 8 \\
\hline FLO & & & 6 & 2 & 5 & & 5 & & 5 & 2 & & & & & 28 & 4 & 64 \\
\hline \multicolumn{18}{|c|}{ Serovar Enteritidis } \\
\hline & MIC & & & & & & & & & & & & & & $\% R$ & $\mathrm{MIC}_{50}$ & $\mathrm{MIC}_{90}$ \\
\hline & $<0.5$ & 0.5 & 1 & 2 & 4 & 8 & 16 & 32 & 64 & 128 & 256 & 512 & 1024 & $>1024$ & & & \\
\hline EEE & 17 & & 1 & 1 & & & & & & & & & & & 5.3 & $<0.5$ & $<0.5$ \\
\hline TET & & & & 1 & 7 & 5 & 3 & & & & 2 & 1 & & & 15.8 & 8 & 256 \\
\hline SXT & & & & 1 & 3 & 4 & & 4 & 5 & & & 1 & 1 & & 10.5 & 32 & 64 \\
\hline CLR & 5 & & 2 & 12 & & & & & & & & & & & 0 & 2 & 2 \\
\hline AMX & & & 14 & 3 & & & & & & 1 & & 1 & & & 10.5 & 1 & 2 \\
\hline FLO & & & 6 & 2 & 5 & & 5 & & 1 & & & & & & 5.3 & 4 & 16 \\
\hline \multicolumn{18}{|c|}{ Serovar Typhimurium } \\
\hline & MIC & & & & & & & & & & & & & & $\% \mathrm{R}$ & $\mathrm{MIC}_{50}$ & $\mathrm{MIC}_{90}$ \\
\hline & $<0.5$ & 0.5 & 1 & 2 & 4 & 8 & 16 & 32 & 64 & 128 & 256 & 512 & 1024 & $>1024$ & & & \\
\hline EEE & & 4 & & 1 & 1 & & & & & & & & & & 33.3 & 0.5 & 4 \\
\hline TET & & & & 1 & & & & 3 & & 1 & & 1 & & & 83.3 & 32 & 512 \\
\hline SXT & & & & & & & & & & & 2 & 4 & & & 100 & 512 & 512 \\
\hline CLR & & & & 6 & & & & & & & & & & & 0 & 2 & 2 \\
\hline AMX & & & & 2 & & 3 & & & & 1 & & & & & 16.7 & 8 & 128 \\
\hline FLO & & & & & & & & & 4 & 2 & & & & & 100 & 64 & 128 \\
\hline
\end{tabular}

Table 3: Percentage distribution of resistance and $\mathrm{MIC}_{50}$ and $\mathrm{MIC}_{90}(\mu \mathrm{g} / \mathrm{mL})$ of $S$ Typhimurium and $S$ Enteritidis serovars.

\begin{tabular}{|c|c|c|c|c|}
\hline Resistance pattern & & $\mathbf{N}$ & Porcine & Equine \\
\hline S. Typhimurium & TET-SXT-FLO & 4 & 2 & 2 \\
\hline S. Enteritidis & TET-SXT-AMX & 1 & 1 & \\
\hline S. Typhimurium & EEE-TET-SXT-FLO & 1 & 1 & \\
\hline S. Typhimurium $y$ S. Enteritidis & EEE-TET-SXT-AMX-FLO & 2 & 2 & \\
\hline
\end{tabular}

TET: Oxytetracycline; SXT: Trimethoprim/sulfamethoxazole; FLO: Florfenicol; AMX: Amoxicillin; EEE: Enrofloxacin.

Table 4: Resistance patterns obtained of the serovars $S$ Typhimurium and $S$ Enteritidis isolated from different animal sources.

31 Salmonella strains were isolated in cattle belonging to 12 different types of serovars, and the transduction of microbial resistance from $S$ Heidelberg to $S$. Typhimurium was demonstrated, phage resistant to multiple beta-lactam antibiotics and tetracycline blaCMY-2, tet (A) and tet (B), respectively [18]. Later on, a 58\% resistance to trimethoprim/ sulfamethoxazole and $56 \%$ to tetracycline, followed by ampicillin and amoxicillin was reported [7]. In Chile, in a preliminary study, $20.5 \%$ of Salmonella strains isolated, mainly from pigs, showed MDR, being oxytetracycline the agent showing the highest resistance (69.1\%) [31]. The results reported here show that serovars isolated from swine reach a high multi-resistance.

It is worth mentioning that the main Salmonella serovars showing multidrug resistance were obtained from clinical samples. It is also important to mention that many of the serovars isolated from seagulls showed an intermediate sensitivity according to the Kirby-Bauer qualitative method of susceptibility, but susceptibility to them will depend on which species will be infected by these serovars. It was reported that 9\% of seagulls (Larus occidentalis) studied, in 2008, in California were carriers of Salmonella but only from one of those seagulls an antibiotic resistant Salmonella was isolated [32]. Subsequently, was reported a $24 \%(\mathrm{n}=216)$ isolation of Salmonella from young blackheaded gulls (Larus ridibundus) being S. Enteritidis (PT 8 and 4) the most prevalent showing a $28 \%$ of resistance to antibiotics [33].

As mentioned above, the effect of antimicrobials in the equine species, with respect to the acquisition of bacterial resistance in humans, has not been reported; however the frequency pattern is repeated for this species. It makes sense, because horses can be infected, by direct or indirect contact, by Salmonella infected feces of several other animal species. Salmonella serovars isolated from horses $(\mathrm{n}=232)$ were predominantly $S$. Typhimurium and the main antibacterial resistance was found for tetracycline and for ampicillin [27]. Most isolates were susceptible to ceftiofur and enrofloxacin. It must be emphasized the diversity of resistance patterns shown in this work $(n=16)$ including antimicrobial susceptibility tests to 9 antimicrobials. Resistance 
patterns included from only tetracycline to different combinations of antimicrobials and it can be concluded that $S$. Typhimurium phage type in horses, 506 (DT 104), corresponds to those found in humans, pigs and cattle for the same time period of this study.

In relation to meat of animal origin intended for human consumption, a high percentage of isolation of various Salmonella serovars from meat samples of pigs and chickens, including only $0.1 \%$ $S$. Typhimurium and only $1.2 \%$ S. enteridis, and a high frequency of antibacterial resistance to at least one antibiotic (78.4\% of the isolates) was reported in North Vietnam [13]. In China, samples obtained from slaughtered chickens and pigs showed $45.2 \%$ and $29.2 \%$ of Salmonella isolation, respectively [34]. The predominant serovar in chickens was $S$. Enteritidis but pigs showed mainly $S$. Typhimurium plus $S$ Derby and $S$. Enteritidis. Highly multidrug resistant Salmonella was also reported in chicken eggs for human consumption [14].

It is important to consider that some studies from around the world have reported ciprofloxacin resistant Salmonella [7], particularly in less frequent serovars [23]. Since this antibiotic is widely used in human salmonellosis it should be included in future epidemiological studies to monitor the development and spread of resistance to this antimicrobial in different countries and regions worldwide. Other studies reported $8.6 \%$ and $10 \%$ ciprofloxacin resistant Salmonella Indiana serovar strains in chickens and pigs, respectively [34].

It is necessary, therefore, to characterize the genotypic resistance of the isolates obtained in this work. For this purpose, the strains are kept in the culture collection of the MedVet-UdeC Laboratory at $-20^{\circ} \mathrm{C}$ for further studies including patterns of genetic resistance to be determined by molecular techniques. Since it is known that the genetic transmission occurs easily among different bacterial species in the gastrointestinal environment [18], it is not unthinkable that this transmission might occur at a high frequency not only within the same animal species, but also between different species, not only production animals but also including wildlife, through the release of the feces of infected animals. It would be also important to maintain a database with the results obtained at different regions of the world in order to monitor or support control programs and food safety in countries not having sufficient information, as compared to the countries of the European Community [8]. It would be also advisable to include the phylogenetic study of Salmonella isolates to know their origins and relationship between different circulating serovars.

Undoubtedly, also it is necessary to determine the prevalence of the genes determining antibiotic resistance in the serovars isolated to assess variations within or between them. Salmonella possess various virulence and antimicrobial resistance mechanisms able to challenge public health strategies. As the understanding of the pathogenicity mechanisms and factors leading to antimicrobial resistance development improves, there is a hope to limit the load of these pathogens to the environment.

It is an important challenge for the anti-Salmonella therapy, especially in humans, the diversity of treatments. Veterinary medicine has a similar challenge because, for example, the treatment for certain animal species involves the use of cephalosporins which are contraindicated for other animal species, finally complicating the treatment. Non-typhoid Salmonella can cause serious septicemic diseases in adults, particularly in immunocompromised individuals having viral diseases making them more susceptible to these infections, which also carry resistance to several antimicrobials and also in very young individuals with viral diseases producing a decrease in the immune response, especially in human medicine in children under 5 years of age.

However, it has been detected in humans a decrease in the frequency of $S$. Typhimurium and $S$. Enteritidis with a concomitant increase of other serovars [12]. Moreover, it is interesting the association, made in Ethiopia, of Salmonella infections in humans with the consumption of raw vegetables, whose contamination may result from animal feces [35]. Since infection outbreaks will vary depending on the serovar involved and its antimicrobial resistance pattern, information of serovars of $S$. enterica circulating in a given geographical area and their antibiotic susceptibility is necessary to design appropriate interventions to control these outbreaks. Thus, the phylogenetic study of the serovars found in the different sources analyzed in the present work becomes relevant, as it is also the case of the genetic study of the predominant serovars in a region.

In conclusion, the control and monitoring of Salmonella serovars from different animal sources is of great importance for human health. The permanent potential risk of these serovars present in feces contaminating the environment and, thus, animal food, constitute the first priority to control infections and to establish antibiotic therapy strategies.

\section{Funding}

Laboratory of Veterinary Microbiology. Department of Pathology and Preventive Medicine, Faculty of Veterinary Sciences, University of Concepcion, Chile.

\section{Acknowledgements}

Authors wish to thank the very special collaboration of Mr. Fredy Riquelme laboratory technician at the MedVet-UdeC Laboratory for his assistance.

\section{References}

1. Martelli F, Davies RH (2012) Review Host adapted serotypes of Salmonella enterica. Salmonella serovars isolated from table eggs: An overview. Food Res Int 45: 745-754.

2. Guibourdenche $M$, Roggentin $P$, Mikoleit $M$, Fields $\mathrm{PI}$, Bockemühl J, et al. (2010) Supplement 2003-2007 (No. 47) to the White-Kauffmann-Le Minor scheme. Res Microbiol 161: 26-29.

3. Scallan E, Griffin PM, Angulo FJ, Tauxe RV, Hoekstra RM (2011) Foodborne illness acquired in the United States--unspecified agents. Emerg Infect Dis 17: 16-22

4. Centers for Disease Control and Prevention (2014) Behavioral Risk Factor Surveillance System (BRFSS).

5. Hohmann EL (2001) Nontyphoidal salmonellosis. Clin Infect Dis 32: 263-269.

6. Zhao S, McDermott PF, White DG, Qaiyumi S, Friedman SL, et al. (2007) Characterization of multidrug resistant Salmonella recovered from diseased animals. Vet Microbiol 123: 122-132.

7. Yang B, Qu D, Zhang X, Shen J, Cui S, et al. (2010) Prevalence and characterization of Salmonella serovars in retail meats of marketplace in Shaanxi, China. Int J Food Microbiol 141: 63-72.

8. De Knegt LV, Pires SM, Hald T (2015) Using surveillance and monitoring data of different origins in a Salmonella source attribution model: a European Union example with challengesd and proposed solutions. Epidemiol Infect 143: 11481165.

9. European Food Safety Authority (EFSA) (2010) The Community Summary report on Trends and sources of zoonoses and zoonotic agents and food-borne outbreaks in the European Union in 2008. EFSA Journal 8: 1546.

10. Laufer AS, Grass J, Holt K, Whichard JM, Griffin PM, et al. (2015) Outbreaks of Salmonella infections attributed to beef -United States, 1973-2011. Epidemio Infect 143: 2003-2013

11. De Knegt LV, Pires SM, Hald T (2015) Attributing foodborne salmonellosis in humans to animal reservoirs in the European Union using a multi-country stochastic model. Epidemiol Infect 143: 1175-1186. 
Citation: López-Martín JI, González-Acuña D, García CA, Carrasco LO (2016) Isolation and Antimicrobial Susceptibility of Salmonella Typhimurium and Salmonella Enteritidis in Fecal Samples from Animals. J Antimicro 2: 109. doi:10.4172/2472-1212.1000109

12. Foley SL, Lynne AM (2008) Food animal-associated Salmonella challenges: pathogenicity and antimicrobial resistance. J Anim Sci 86: E173-187.

13. Thai, TH, Hirai T, Lan NT, Yamaguchi R (2012) Antibiotic resistance pro? les of Salmonella serovars isolated from retail pork and chicken meat in North Vietnam. International Journal of Food Microbiology 156: 147-151.

14. Singh S, Yadav AS, Singh SM, Bharti $P$ (2010) Prevalence of Salmonella in chicken eggs collected from poultry farms and marketing channels and their antimicrobial resistance. Food Research International 43: 2027-2030.

15. Moosavy M, Esmaeili S, Amiri FB, Mostafavi E, Salehi TZ (2015) Detection of Salmonella spp in commercial eggs in Iran. Iran J Microbiol 7: 50-54.

16. Minette HP (1986) Salmonellosis in the marine environment. A review and commentary. Int J Zoonoses 13: 71-75.

17. Kruse $\mathrm{H}$, kirkemo AM, Handeland $\mathrm{K}$ (2004) Wildlife as source of zoonotic infections. Emerg Infect Dis 10: 2067-2072.

18. Zhang $Y$, LeJeune $J$ (2008) Transduction of blaCMY-2, tet(A), and tet(B) from Salmonella enterica subspecies enterica serovar Heidelberg to $S$ Typhimurium. Vet Microbiology 129: 418-425.

19. Kariuki S, Onsare RS (2015) Epidemiology and Genomics of Invasive Nontyphoidal Salmonella Infections in Kenya. Clin Infect Dis 61: S317-324.

20. de Oliveira DS, Siqueira F, Ruschel L, Brandelli A (2005) Antimicrobial resistance in Salmonella Enteritidis strains isolated from broiler carcasses, food, human and poultry-related samples. International J of Food Micro 97: 297-305.

21. Rodriguez-Rivera LD, Wright EM, Siler JD, Elton M, Cummings KJ, et al. (2014) Subtype analysis of Salmonella isolated from subclinically infected dairy cattle and dairy farm environments reveals the presence of both human- and bovineassociated subtypes. Vet Microbiol 170: 307-316.

22. Haley CA, Dargatz DA, Bush EJ, Erdman MM, Fedorka-Cray PJ (2012) Salmonella prevalence ans antimicrobial susceptibility from the National Animal Health Monitoring System swine 2000 and 2006 studies. Journal of Food Protection 75 : 428-436.

23. Münch S, Braun P, Wernery U, Kinne J, Pees M, et al. (2012) Prevalence, serovars, phage types, and antibiotic susceptibilities of Salmonella strains isolated from animals in the United Arab Emirates from 1996 to 2009. Trop Anim Health Prod 44: 1725-1738.

24. Clinical and Laboratory Standards Institute (CLSI) (2009) Performance standards for antimicrobial disk susceptibility test; approved standard. M02-A10: 29.
25. López-Martín J, Junod T, Riquelme F, Contreras C, González-Acuña D (2011) Detection of Salmonella and Mycobacterium species in Dominican gulls ( Larus dominicanus ) and gulls Franklin ( Franklin's Gull ) in the city of Talcahuano , Chile. Rev. Med Chile 139: 1496-1502.

26. Refsum T, Vikøren T, Handeland K, Kapperud G, Holstad G (2003) Epidemiologic and pathologic aspects of Salmonella Typhimurium infection in passerine birds in Norway. J Wildl Dis 39: 64-72.

27. van Duijkeren E, Wannet WJB, Heck MEOC, van Pelt W, van OldruitenborghOosterbaan SMM, et al. (2002) Sero types, phage types and antibiotic susceptibilities of Salmonella strains isolated from horses in The Netherlands from 1993 to 2000. Vet Micr 86: 203-212.

28. Graham JP, Boland JJ, Silbergeld E (2007) Growth promoting antibiotics in food animal production: an economic analysis. Public Health Rep 122: 79-87.

29. Esaki H, Morioka A, Ishihara K, Kojima A, Shiroki S, et al. (2004) Antimicrobia susceptibility of Salmonella isolated from cattle, swine and poultry (20012002): report from the Japanese Veterinary antimicrobial resistance monitoring program. Journal of Antimicrobial Chemotherapy 53: 266-270.

30. Tanaka T, Imai Y, Kumagae N, Sasaki T, Ochiai N, et al. (2014) Quantitative microbiological evaluation of Salmonella Typhimurium shed in diarrhea, loose and normal stools of infected pigs. Open Journal of Veterinary Medicine 4: 5866.

31. Junod T, López-Martin J, Gädicke P (2013) Antimicrobial susceptibility of animal and food isolates of Salmonella enterica. Rev Med Chil 141: 298-304.

32. Stoddard RA, DeLong RL, Byrne BA, Jang S, Gulland FM (2008) Prevalence and characterization of Salmonella spp. among marine animals in the Channel Islands, California. Dis Aquat Organ 81: 5-11.

33. Dolejská M, Bierosová B, Kohoutová L, Literák I, Cizek A (2009) Antibioticresistant Salmonella and Escherichia coli isolates with integrons and extendedspectrum beta-lactamase in surface water and sympatric black-headed gulls. J Applied Microbiology 106: 1941-1950.

34. Bai L, Lan R, Zhang X, Cui S, Xu J, et al. (2015) Prevalence of Salmonella Isolates from Chicken and Pig Slaughterhouses and Emergence of Ciprofloxacin and Cefotaxime Co-Resistant S. enterica Serovar Indiana in Henan, China. PLoS One 10: e0144532.

35. Eguale T, Gebreyes WA, Asrat, Alemayehu H, Gunn JS, et al. (2015) Nontyphoidal Salmonella serotypes, antimicrobial resistance and co-infection with parasites among patients with diarrhea and other gastrointestinal complaints in Addis Ababa, Ethiopia. BMC Inf Dis 15: 497. 